\title{
Effects of Si Addition on Microstructure, Properties and Serration Behaviors of Lightweight Al-Mg-Zn-Cu Medium-entropy Alloys
}

\author{
Yasong Li, Ruixuan Li, Yong Zhang* \\ State Key Laboratory for Advanced Metals and Materials, University of Science and Technology Beijing, Beijing 100083, China
}

*Corresponding Author: Yong Zhang, 30\# Xueyuan Road, Beijing, China; drzhangy@ustb.edu.cn

\begin{abstract}
:
A series of as-cast lightweight multicomponent alloys $\mathrm{Al}_{(86-\mathrm{x})} \mathrm{Mg}_{10} \mathrm{Zn}_{2} \mathrm{Cu}_{2} \mathrm{Si}_{\mathrm{x}}(\mathrm{x}=0,0.3,0.6,0.9,1.2$ at.\%) were prepared by a vacuum induction furnace with a steel die. With the addition of $\mathrm{Si}$, the reticular white $\mathrm{Al}-\mathrm{Cu}$ phase deposited were gradually replaced by the gray eutectic $\mathrm{Mg}$-Si phase, while the compressive strength of the alloys increases first and then decreases slowly. It is particularly noteworthy that the compression plasticity also exhibits this trend. When the Si content is 0.9 at.\%, the compressive strength reaches its maximum at $779.11 \mathrm{MPa}$ and the compressive plasticity reaches $20.91 \%$. The effect of the addition of $\mathrm{Si}$ on the serration behavior of alloy was also studied; we found that the addition of $\mathrm{Si}$ introduces a new MgSi phase, and with the change of Si is significantly affects the morphology of the precipitated phase, which affects the serration behavior of the alloys. The comprehensive mechanical properties of the alloy are optimal at the critical point where the serration behavior disappears. In this work, we have provided a method and a composition for the preparation of a low-cost, high-strength, lightweight medium-entropy alloys.

Keywords: Si Addition; Microstructure and Properties; Serration Behavior; Lightweight, Medium-entropy Alloys

Citation: Y.S. Li, R.X. Li, Y. Zhang., Effects of Si Addition on Microstructure, Properties and Serration Behaviors of Lightweight Al-Mg-Zn-Cu Medi-um-entropy Alloys. Res Appl Mat Sci, 2019,1(1): 10-17. https://doi.org/10.33142/msra.v1i1.666
\end{abstract}

\section{Introduction}

As the development of the human beings, environmental issues and energy crisis are gradually being social cognitions. Energy saving, environmental protection, and the low-carbon life have become indispensable topics in people's daily life. Enhancing the use of light-weight materials can effectively save energy and reduce consumption. Besides, traditional aluminum/magnesium alloys have shown good specific stiffness and specific strength, meanwhile, a large number of research results and corresponding applications have been obtained ${ }^{[1-6]}$. In recent years, the high-entropy alloys have become a hot issue for researchers. Conventional high-entropy alloys are generally formed by Co, $\mathrm{Cr}, \mathrm{Fe}, \mathrm{Ni}, \mathrm{Mn}$ as basic elements with alloying elements such as $\mathrm{Al}, \mathrm{Cu}, \mathrm{Ti}$, and $\mathrm{V}$ et al. adding to the solid solution alloys ${ }^{[7-10]}$. These alloys exhibit excellent mechanical properties such as high strength, high toughness ${ }^{[9]}$, good wear resistance ${ }^{[11]}$, and corrosion resistance ${ }^{[12,13]}$. However, such alloys tend to have high density, therefore, how to develop lightweight and low cost high-entropy materials have become the focus of many researchers.

There have been some lightweight high entropy alloys with great properties developed resent years. A new type of low-den- sity, high-entropy alloy $\mathrm{Al}_{20} \mathrm{Li}_{20} \mathrm{Mg}_{10} \mathrm{Sc}_{20} \mathrm{Ti}_{30}$ has developed by Khaled M. Youssef et al. ${ }^{[14]}$. This kind of alloy exhibit a nanoscale single face-centered cubic (FCC) solid solution phase, and due to the ultrafine grain structure, it shows not only excellent hardness similar to ceramics such as $\mathrm{SiC}$, but also better ductility. However, since the melting point of Sc and Ti are much higher than $\mathrm{Al}, \mathrm{Li}$, and $\mathrm{Mg}$, with mechanical alloying method the problem of preparing such alloys were solved. Furthermore, a new hexagonal close-packed (HCP) structure lightweight high-entropy alloys $\mathrm{Al}_{20} \mathrm{Be}_{20} \mathrm{Fe}_{10} \mathrm{Si}_{15} \mathrm{Ti}_{35}$ are prepared by TSENG KoKai et al. ${ }^{[15]}$, which exhibit excellent mechanical properties at both room temperature and high temperature. Rui Feng et al. ${ }^{[16]}$ studies the AlCrFeMnTi high-entropy alloys by means of simulation calculation, and the design ideas of lightweight high-entropy alloys are revealed from the perspective of phase diagram, however, due to the addition of $\mathrm{Cr}, \mathrm{Fe}, \mathrm{Mn}$, the density of the so-called lightweight material is still high, and the design of lightweight high-entropy alloy has not been fully revealed. Xing-hao Du et al. ${ }^{[17]}$ reports a kind of lightweight high-entropy alloys prepared through copper die casting, and it is found that they have superior specific strength with a tetragonal symmetry lattice, however the plasticity of the alloy is not mentioned. Additionally, Rui Li 
et al. ${ }^{[18,19]}$ investigated $\mathrm{Mg}_{x}(\mathrm{MnAlZnCu})_{100-x}$ lightweight high-entropy alloys are and they exhibit high hardness, but the comprehensive mechanical properties are very poor.

More recently, many scholars have also conducted related researches and experiments. Since $\mathrm{Al}, \mathrm{Li}, \mathrm{Mg}$ are common lightweight structural material elements, Yang et al. ${ }^{[20]}$ consider and develop two systems of AlLiMgZnCu and AlLiMgZnSn using the design principles of high-entropy alloys. However, in view of the strong electronegativity of these elements, the high entropy effects do not promote the formation of the single phase solid solution, while bulk intermetallic compounds are generated instead, which therefore results in deterioration in mechanical properties. On the other hands, When the content of $\mathrm{Al}$ is dominant as 80 at. \%, the alloy tends to form a single face-centered cubic (FCC) solid solution phase. Therefore, two systems of medium-entropy alloy systems $\mathrm{Al}_{80} \mathrm{Li}_{5} \mathrm{Mg}_{5} \mathrm{Zn}_{5} \mathrm{Cu}_{5}$ and $\mathrm{Al}_{80} \mathrm{Li}_{5} \mathrm{Mg}_{5} \mathrm{Zn}_{5} \mathrm{Sn}_{5}$ have been developed, with compressive strengths exceeding $800 \mathrm{MPa}$ and compression plasticity exceeding $17 \%$. It can be found that although the mixing entropies of these alloys did not reach the maximum, they all showed superior mechanical properties. Based on this research, Baek Eun-Ji, et al. ${ }^{[21]}$ develop an alloy system as $\mathrm{Al}_{70} \mathrm{Mg}_{10} \mathrm{Si}_{10} \mathrm{Zn}_{5} \mathrm{Cu}_{5}$ by means of ultrasonic melting technology, and study the evolution law of the heat treatment phase of the alloys. In addition, the solid solution of medium-entropy alloy $\mathrm{Al}-6 \mathrm{Mg}-9 \mathrm{Si}-10 \mathrm{Cu}-10 \mathrm{Zn}-3 \mathrm{Ni}$ (wt. $\%)$ alloy and phase evolution at different aging temperatures and times were also studied ${ }^{[22,23]}$, however, ultrasonic melt degassing technology can significantly improve the mechanical properties of the alloy, but the mechanical properties of the alloy at room temperature are not good under high Si content, and it is difficult to decrease the size and morphology of the Si-rich precipitate by heat treatment. Li et al. ${ }^{[24]}$ use supergravity investigated the microstructures of the lightweight $\mathrm{Al}-\mathrm{Li}-\mathrm{Mg}-\mathrm{Zn}-\mathrm{Cu}$ alloy, it shows that the supergravity method can achieve performance-enhanc- ing alloys by centrifugation in a short time, gravity is also an entropy force, paving the way for the design and synthesis of entropy alloys with intriguing properties.

In this study, the low cost lightweight $\mathrm{Al}_{(86-x)} \mathrm{Mg}_{10} \mathrm{Zn}_{2} \mathrm{Cu}_{2} \mathrm{Si}_{x}$ $(x=0,0.3,0.6,0.9,1.2$ at. \%) medium-entropy alloys were prepared. The effect of different Si additions on the microstructure and properties of the alloy was investigated with SEM, universal testing machine, and micro hardness tester. At the same time, the serrated flow behaviors on the compressive stress-strain curve of the alloys were also studied. The effect of Si addition on the serrated flow behaviors and the effect of serrated flow behaviors on the mechanical properties of the alloys were also discussed.

\section{Materials and Methods}

The alloy ingots of about $120 \mathrm{~g}$ are prepared by an Ar gas-induced vacuum induction furnace, using industrial pure aluminum (99.7 wt. \%), pure magnesium (99.9 wt. \%), pure zinc (99.9 wt. $\%)$, pure copper(99.9 wt. \%) and pure poly-silicon (99.9 wt. \%). They were melt at $800^{\circ} \mathrm{C}$ for 15 minutes with induction stirring, then poured into a $30 \mathrm{~mm}$ diameter cylindrical steel die. The nominal compositions of the alloys are shown in Table 1.

The microstructures are observed by Zeiss SUPRA 55 field emission scanning electron microscope (SEM) with energy dispersive spectroscopy (EDS) and electron backscattered diffraction (EBSD). In order to observe the total precipitate phase morphology better, the alloys structure were pre-etched with Keller reagent for $15 \mathrm{~s}$. The grain sizes of alloys were analyzed using the EBSD, and the compositions of the precipitated phase were analyzed by EDS.

This experiment uses (Rigaku) D/MAX-RB X-ray diffract-meter to characterize the phase composition of different $\mathrm{Si}$ content alloys. The experimental conditions are $\mathrm{Cu}$ target $\mathrm{Ka}$, working voltage $30 \mathrm{kV}$, working current $100 \mathrm{~mA}$, scanning speed 10 degree $/ \mathrm{min}$, range $10-90^{\circ}$.

Table 1. The elemental composition $(120 \mathrm{~g} \pm 0.3 \%)$

\begin{tabular}{|c|c|c|c|c|c|}
\hline No. & $\mathrm{Al}$ & $\mathrm{Mg}$ & $\mathrm{Zn}$ & $\mathrm{Cu}$ & $\mathrm{Si}$ \\
\hline Al86Mg10Zn2Cu2 & 99.1 & 10.5 & 5.6 & 5.4 & 0 \\
\hline Al85.7Mg10Zn2Cu2Si0.3 & 98.8 & 10.2 & 5.6 & 5.4 & 0.4 \\
\hline Al85.4Mg10Zn2Cu2Si0.6 & 98.2 & 10.2 & 5.6 & 5.4 & 0.8 \\
\hline Al85.1Mg10Zn2Cu2Si0.9 & 97.9 & 10.2 & 5.5 & 5.5 & 1.1 \\
\hline Al84.8Mg10Zn2Cu2Si1.2 & 97.5 & 10.2 & 5.6 & 5.4 & 1.5 \\
\hline
\end{tabular}
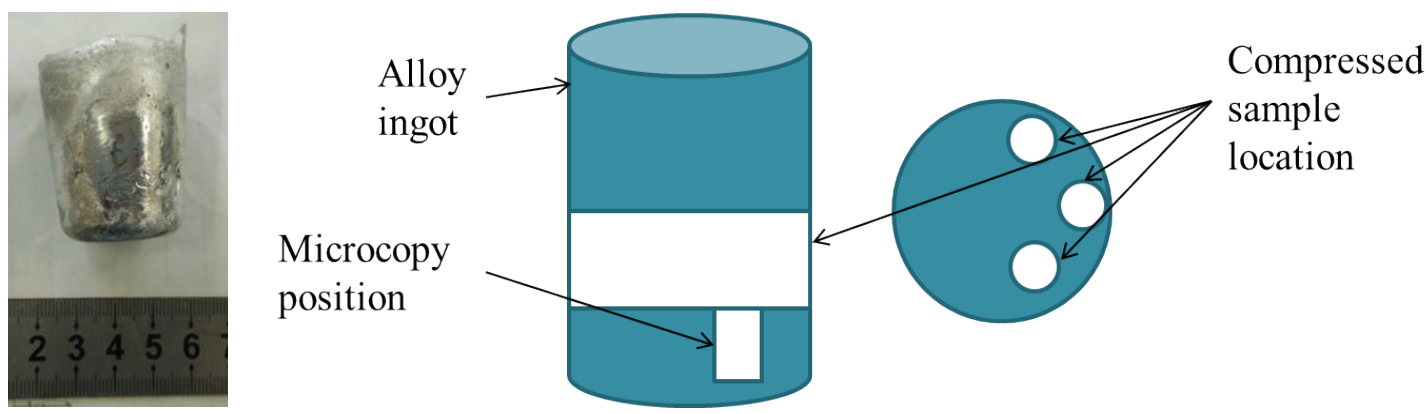

Figure 1 The alloy ingot and the sampling position of the metallographic sample of the compressed sample 
The experiments use CMT 4305 Universal Testing Machine to test the compression mechanical properties at room temperature. Using the HXD-1000TM micro-hardness tester to detect the Vickers micro-hardness of the alloy. The alloy ingot and the sampling position of the metallographic sample of the compressed sample are shown in Figure 1.

\section{Results and Discussion}

\subsection{Alloy design}

As an inexpensive and lightweight semi-metal element, Si element is added to the aluminum alloy to improve the mechanical properties of the alloy ${ }^{[2,5,25-27]}$. Since $\mathrm{Mg}$ and $\mathrm{Si}$ are liable to form a high melting point intermetallic compound, for traditional cast aluminum alloys, the $\mathrm{Mg} / \mathrm{Si}$ ratio has always been strictly controlled variable ${ }^{[28,29]}$. Eun-Ji Baek et al. ${ }^{[21]}$ showed that two kinds of Si-containing phases, $\mathrm{Mg}_{2} \mathrm{Si}, \mathrm{Si}$ and $\mathrm{Al}_{5} \mathrm{Cu}_{2} \mathrm{Mg}_{8} \mathrm{Si}_{6}, \mathrm{Si}$ were formed in the $\mathrm{Al}_{70} \mathrm{Mg}_{10} \mathrm{Si}_{10} \mathrm{Cu}_{5} \mathrm{Zn}_{5}$ multi-component alloy, during the solidification of the alloy, a stable $\mathrm{Mg}_{2} \mathrm{Si}$ phase is primary precipitated at $514-591{ }^{\circ} \mathrm{C}$, when the temperature rises to $500-524^{\circ} \mathrm{C}$, the Si particles precipitated, while the $\mathrm{Al}_{5} \mathrm{Cu}_{2} \mathrm{Mg}_{8} \mathrm{Si}_{6}$ phase is formed at $440{ }^{\circ} \mathrm{C}$. Furthermore, with the addition of $\mathrm{Mg}, \mathrm{Zn}$ and $\mathrm{Cu}$, some other intermetallics such as $\mathrm{Mg}-\mathrm{Zn}$ and $\mathrm{q}-\mathrm{Al}_{2} \mathrm{Cu}$ are formed. As these precipitates have lower melting point, they finally formed out ${ }^{[1,20,30,31]}$. In addition, Shao et al. ${ }^{[32]}$ studied the high-entropy alloys of AlMgZnCuSi system, which obtained the best mechanical properties of $\mathrm{Al}_{85} \mathrm{Mg}_{10.5} \mathrm{Zn}_{2.025} \mathrm{Cu}_{2}$ ${ }_{025} \mathrm{Si}_{0.45}$ alloy. Therefore, this paper intends to study the changes of microstructure and properties of $\mathrm{Al}_{86} \mathrm{Mg}_{10} \mathrm{Zn}_{2} \mathrm{Cu}_{2}$ alloy under the condition of lower Si addition.

\subsection{Effect of Si addition on microstructure}

Figure 2 shows the SEM-AsB photos of $\mathrm{Al}_{(86-x)} \mathrm{Mg}_{10} \mathrm{Zn}_{2} \mathrm{Cu}_{2} \mathrm{Si}_{x}$ alloys under different $\mathrm{Si}$ addition, in which we can found that, with the addition of $\mathrm{Si}$, the white eutectic network precipitate is replaced by the black precipitated phase. Figure 2(a) shows the microstructure of the $\mathrm{Al}_{86} \mathrm{Mg}_{10} \mathrm{Zn}_{2} \mathrm{Cu}_{2}$ alloy, with only white eutectic phase appearing, and with the addition of Si, a black strip phase appears, partially replacing the white eutectic network precipitate, which are shown in Figure 2(b). When the Si content reaches 0.6 at. $\%$, the small strip of black precipitated phase converted into the gray eutectic morphology, as shown in Figure 2(c). As the Si content reaches 0.9 at. \%, the black granular phase and the gray eutectic morphology become obviously larger, as shown in Figure 2(d). When the addition of Si reaches 1.2at. \%, the black granular phases gradually increase, and a larger area of eutectic structure appears Figure 2(e). Therefore, a large area of eutectic structure can be formed in the alloy microstructure by the addition of a trace amount of $\mathrm{Si}$, so that the casting property of the alloy can be optimized accordingly. The Figure 2(f) shows the electron backscattered diffraction (EBSD) Euler picture of the alloy with the addition Si 0.9at. \%, we can find that the grain size of the alloy is between $200-300 \mathrm{~mm}$, since the lattice parameters of the $\mathrm{B}, \mathrm{C}$, and $\mathrm{D}$ phases cannot be determined, they were appeared as black blind spots on the EBSD image. Also these phases were not only at the grain boundary, and also exist inside the grains.
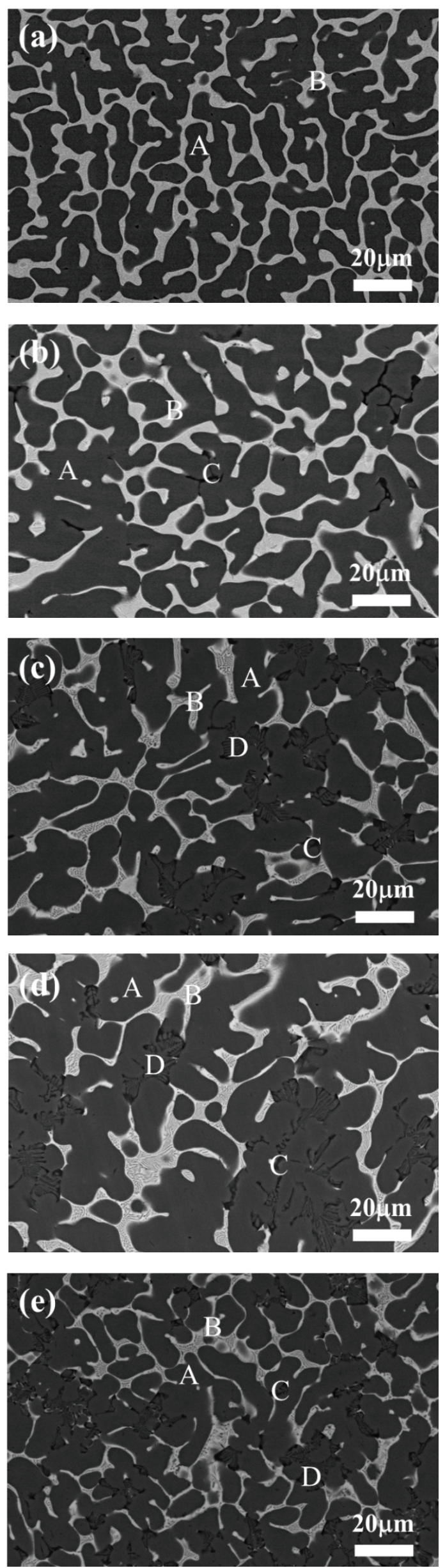


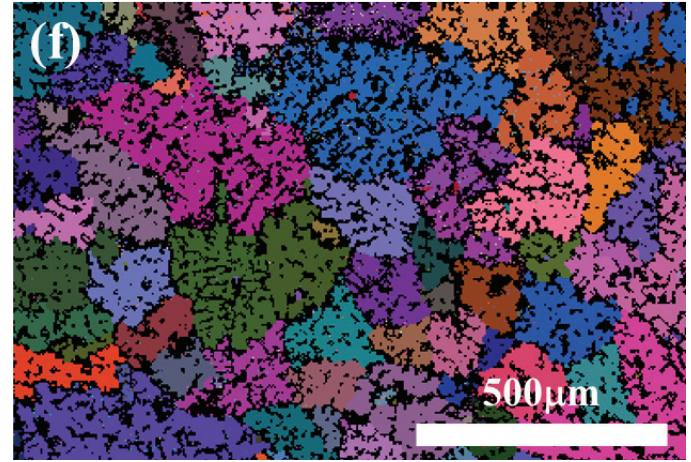

Figure 2 the SEM-AsB photos and EBSD image of the alloys with different $\mathrm{Si}$ addition

\subsection{Effect of phase formation}

Figure 3 shows the XRD pattern of the alloys under different $\mathrm{Si}$ addition conditions. XRD pattern analysis of the alloys exhibit that the peak of $\mathrm{Mg}_{2} \mathrm{Si}$ appears and become stronger when the amount of Si reaches 0.6at. \%, and the $\mathrm{Mg}_{32}(\mathrm{AlZn})_{49}$ weakened or even disappeared meanwhile. Eun-Ji Baek's ${ }^{[21]}$ research shows that three kinds of $\mathrm{Si}$-containing precipitates are produced in $\mathrm{Al}_{70} \mathrm{Mg}_{10} \mathrm{Si}_{10} \mathrm{Cu}_{5} \mathrm{Zn}_{5}$. Their results also show that the Si-rich precipitates tend to have higher melting points, and results in the Si-rich precipitates preferentially precipitated during the solidification process, thereby suppressing the precipitation of the MgCuZn-rich phase.

The compositions of different precipitates are characterized by SEM with EDS, which is shown in Table 2. The EDS analysis has shown that a new $\mathrm{Si}$-rich precipitate phase is formed in the alloy with minute quantity $\mathrm{Si}$, and the microstructures have changed significantly. We can see from the EDS data analysis that the three precipitates in the alloy contain different elemental differences. The alloy without $\mathrm{Si}$ addition are formed by matrix A phase and B phase. The matrix A phase is $\alpha$-Al solid solution phase, and it contains less solute elements. The $\mathrm{B}$ phase is a eutectic phase, which is rich of $\mathrm{Mg}-\mathrm{Cu}-\mathrm{Zn}$ elements. The $\mathrm{C}$ phase is possibly the $\mathrm{Mg}_{2} \mathrm{Si}$ phase, and D is the eutectic $\mathrm{Mg}_{2} \mathrm{Si}$.

Table 2. The energy dispersive spectroscopy(EDS) analysis

\begin{tabular}{ccccccc}
\hline \multicolumn{2}{c}{ Element at.\% } & $\mathrm{Al}$ & $\mathrm{Mg}$ & $\mathrm{Zn}$ & $\mathrm{Cu}$ & $\mathrm{Si}$ \\
\hline \multirow{2}{*}{0} & $\mathrm{~A}$ & 92.96 & 5.34 & 1.05 & 0.65 & -- \\
& $\mathrm{B}$ & 67.49 & 20.94 & 4.70 & 6.87 & -- \\
\hline \multirow{2}{*}{ Si0.3 } & $\mathrm{A}$ & 89.37 & 5.98 & 2.81 & 1.84 & -- \\
& $\mathrm{B}$ & 55.54 & 18.13 & 10.59 & 15.52 & -- \\
& $\mathrm{C}$ & 46.45 & 27.68 & -- & 1.46 & 24.42 \\
\hline \multirow{5}{*}{ Si 0.6 } & $\mathrm{A}$ & 92.47 & 4.20 & 2.34 & 0.99 & -- \\
& $\mathrm{B}$ & 53.74 & 16.12 & 11.41 & 18.72 & -- \\
& $\mathrm{C}$ & 67.87 & 14.80 & 1.07 & 1.07 & 15.19 \\
& $\mathrm{D}$ & 72.73 & 17.50 & 0.90 & 0.53 & 8.33 \\
\hline & $\mathrm{A}$ & 91.89 & 4.15 & 2.67 & 1.28 & -- \\
Si 0.9 & $\mathrm{B}$ & 61.74 & 11.68 & 9.96 & 16.63 & -- \\
& $\mathrm{C}$ & 53.99 & 27.50 & 1.10 & 1.27 & 16.15 \\
& $\mathrm{D}$ & 76.78 & 9.73 & 2.95 & 1.84 & 8.71 \\
\hline
\end{tabular}

\begin{tabular}{|c|c|c|c|c|c|c|}
\hline \multicolumn{2}{|c|}{ Element at.\% } & $\mathrm{Al}$ & $\mathrm{Mg}$ & $\mathrm{Zn}$ & $\mathrm{Cu}$ & $\mathrm{Si}$ \\
\hline \multirow{4}{*}{ Si 1.2} & A & 91.24 & 3.96 & 2.95 & 1.85 & -- \\
\hline & B & 49.26 & 18.18 & 12.16 & 20.40 & -- \\
\hline & $\mathrm{C}$ & 51.41 & 23.67 & 1.87 & 0.93 & 22.12 \\
\hline & $\mathrm{D}$ & 73.83 & 14.63 & 2.43 & 1.16 & 7.92 \\
\hline
\end{tabular}
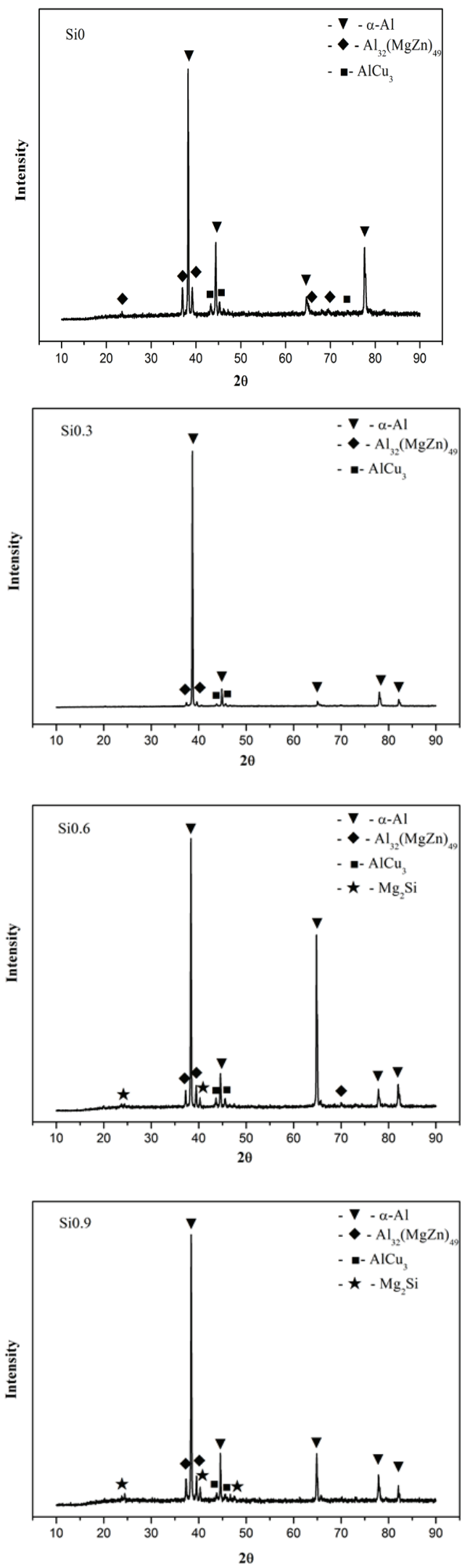


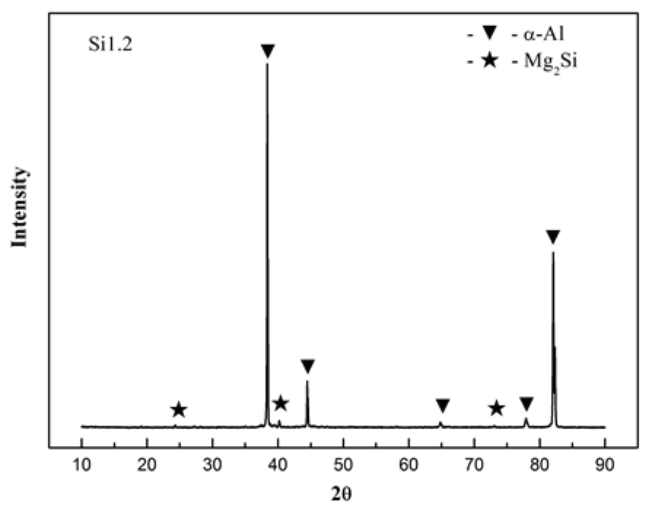

Figure 3 the XRD patterns under different Si contents

\subsection{Properties}

The compressive stress-strain curves and the micro-hardness of the alloys of the alloys are shown in Figure 4. With the addition of $\mathrm{Si}$ addition, the compressive strength of the alloys increases first and then decreases, and its maximum compressive strength reaches $779.11 \mathrm{MPa}$; meanwhile, the compression plasticity of the alloy also shows this trend, and its maximum value reaches $20.9 \%$ when the addition of Si reaches 0.9 at.\%. In addition, it is found that there are serrated flows in the compression process, while when the Si content exceeds 0.9at.\%, the serrated flow disappears, with the compressive strength and plasticity of the alloy decreasing. We will discuss this phenomenon in Section 4.2. We can find that the trend of the micro-hardness of alloys change is similar to the compressive strength of the alloy with Si addition.

\subsection{Serration behavior}

The serrated flow behavior of the alloys has become a hot topic for a long time, typically in superalloys ${ }^{[33]}$, aluminum-magnesium alloys ${ }^{[33,34]}$ and stainless steels ${ }^{[35]}$, in recent years, these behaviors were also found in bulk metal glass ${ }^{[36,37]}$ and high-entro
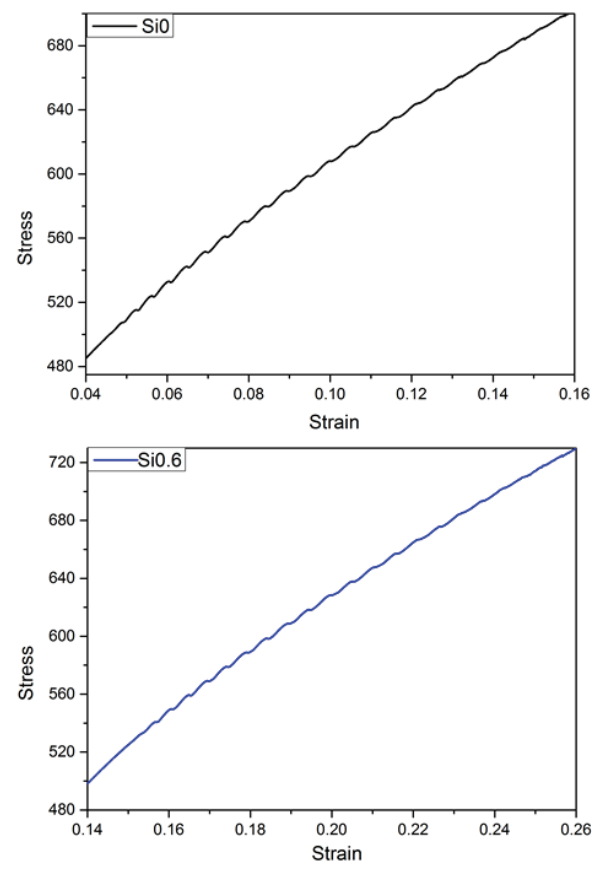
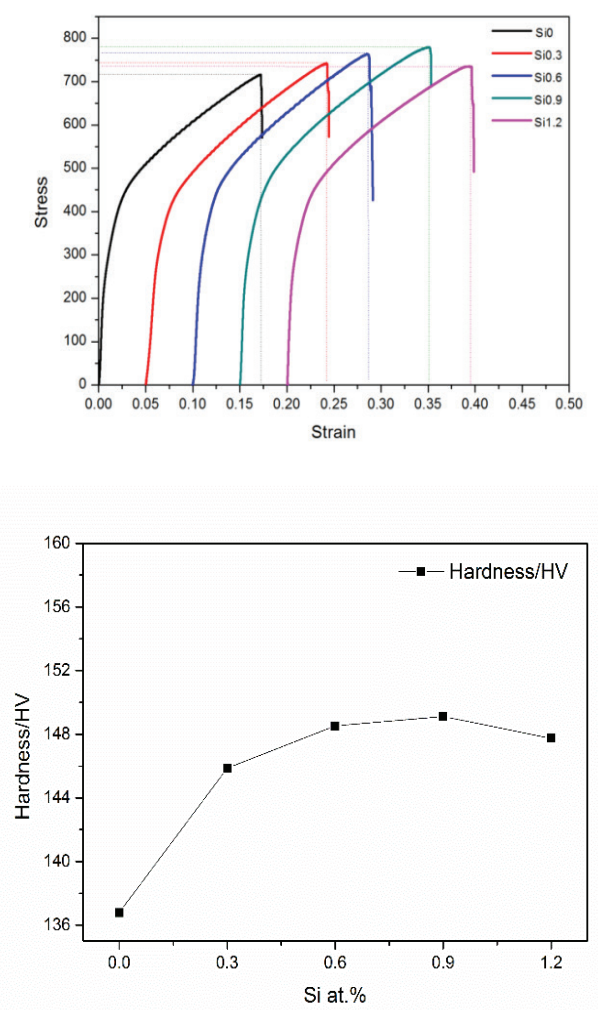

Figure 4 the compressive stress-strain curves and the micro-hardness of the alloys with different Si addition

py alloys ${ }^{[38-40]}$. For these materials, when there is an appropriate temperature, strain rate or pre-deformation, the avalanche shear deformation accompanying the stress zigzag drop will happen during the compression, which is called the Portevin-Le Chat-

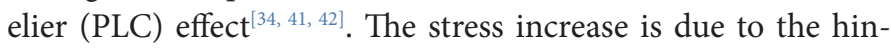
dered and pinned dislocations by the interstitial atoms, which
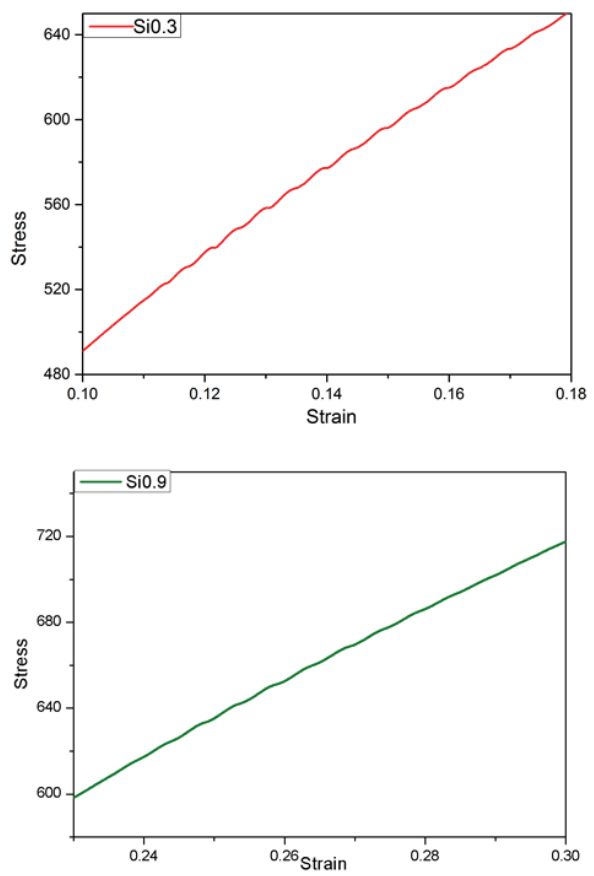

Figure5 the serration flow behavior under initial conditions with different Si addition 
causes the dislocation walls or dislocation loops forming, however, when the stress continues to increase, the dislocations will break away from the bond and continue to slip, so that the stress decrease a little. In this way, the cyclic fluctuation of the stress occurs, which can be seen that the serration behavior yield in the metal crystal is related to the dislocation motion and the extension of the Lüders bands. In aluminum-magnesium alloys, these serration flow behaviors often explained by the dynamic strain aging (DSA) theory which is proposed by Cottrell ${ }^{[43]}$, this theory clarifies the dynamic interaction between movable dislocations and solute atoms. At present, there is some controversy about the interpretation of the theory. The DSA theory focuses on the interaction between solute atoms and defects in the crystal. However, the influence of precipitation in the material on the Portevin-Le Chatelier (PLC) effect cannot be ignored, especially in high alloyed aluminum alloy. As an alloying element in $\mathrm{Al}-\mathrm{Mg}$ alloys, $\mathrm{Si}$ is easy to form $\mathrm{Mg}_{2} \mathrm{Si}$ precipitates with $\mathrm{Mg}$ in the alloys. Therefore, the addition of trace amounts of Si will have a more complicated effect on the serration behavior.

Figure 5 shows the serration behaviors of the alloys, which have $\mathrm{Si}$ addition below $0.9 \mathrm{at} . \%$. It can be found that, with the increase of Si content, the serration behaviors become weaker and then they turns stronger but not stronger than before. However, when the addition of Si reaches 0.9at.\%, Stress-strain curve tends to be smooth. At this time, the alloy exhibits excellent mechanical properties, and the serrated flow is the type $\mathrm{D}^{[34]}$. Through EDS analysis, we can see that the content of solid solution elements in the matrix have a little change. It shows that the change of solid solution elements in the matrix have no obvious influence on the serration flow behavior of alloys. This indicated that the second phases have an effect on the serration flow behavior of alloys.

In order to better study the serration behaviors, we studied the relationship between stress drop with different Si content, which is shown in Figure 6. It can be found that with the increase of strain, the stress drop of the different alloys are shown an approximately linear decrease with slight data fluctuations. On the other hands, with the increase of $\mathrm{Si}$ content, the average value of stress drops first decrease, then increase, and finally turn to 0 when the addition of $\mathrm{Si}$ reaches 0.9 at.\%. This trend is consistent with the serration behavior on the compressive stress-strain curve of the alloys.

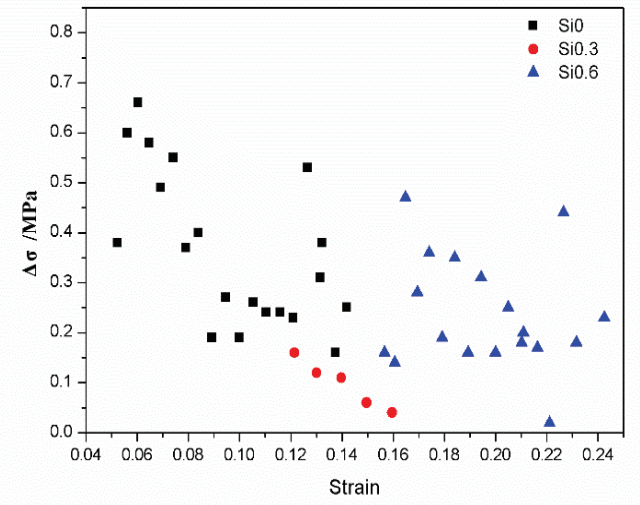

Figure 6 the variation of stress drop with strain under different Si content conditions
Through the analysis of serrated flow behaviors of different alloys, it can be seen that the mechanical properties of the alloys are closely related to the serrated phenomenon. Therefore, the changes of the serrated flow behaviors is mainly due to the changes of the $\mathrm{Mg}$ content, which can be explained by the DSA theory. According to the Cottrell-Bilby type kinetics ${ }^{[43]}$, the solute atom concentration around the dislocation line satisfies Equation 1.

$$
c_{s}=c_{m}\left[1-\exp \left(-p t^{\theta}\right)\right]
$$

where $c_{s}$ is the solute atomic concentration, $c_{m}$ is its saturation value, $t$ is the effective time for the solute atoms to disperse into dislocations ( $t_{w}$ is the waiting time), $p$ is a constant related to the solute atom's ability to diffuse, and $\theta$ is a constant as $1 / 3$ when solute atoms diffuse by tube diffusion. Based on this, when the content of $\mathrm{Mg}$ atoms is lowered, the solute atoms for diffusion are reduced, and the pinning effect on dislocations is lowered. With the addition of $\mathrm{Si}$, a new $\mathrm{Mg} 2 \mathrm{Si}$ precipitate phase partially replaces the $\mathrm{Al}-\mathrm{Mg}-\mathrm{Cu}$ precipitates in the alloy. Due to the precipitation of $\mathrm{Mg} 2 \mathrm{Si}$, which affects the serrated flow behaviors exactly, which causes the serrated flow become weaken. At the same time, the precipitation phase formed by the lower addition of $\mathrm{Si}$ is finer and its distribution in the matrix also lead to an increase in the mechanical properties of the alloy. Therefore, the influencing factor of the serrated flow behaviors in the alloy

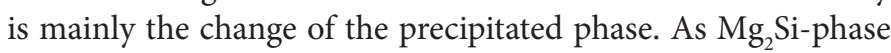
is formed, the other precipitates decrease. As a result, the influence of precipitation on dislocations becomes weaken, which in turn leads to the weaken of the serration behaviors. When the $\mathrm{Si}$ content reaches 0.6 at.\%, the precipitation phase of $\mathrm{Mg}_{2} \mathrm{Si}$ in the alloy increases remarkably, and its corresponding morphology also changes with the content of $\mathrm{Mg}$ in the matrix decreases through EDS analysis. However, the pinning and hindrance of the formed relative dislocations is enhanced, which cause the enhanced strength and serration behavior. As the addition of $\mathrm{Si}$ reaches 0.9 at.\%, a large number of lamellar eutectic structures forms, and the formation of larger granular $\mathrm{Mg}_{2} \mathrm{Si}$ phases leads to further enhancement of alloy strength. Due to the lamellar eutectic structure, the dislocations can easily slides during the compression process, which causes the serration behavior of the alloy to be weaken, and it is very obvious in the compressive stress and strain curve of the alloys. At this time, the stress-strain curve of the alloy has approached the smooth critical point, which may also be the reason for the best compression strength and compression plasticity of the alloy during compression. When the addition amount of Si reaches 1.2at.\%, a larger area of eutectic $\mathrm{Mg} 2 \mathrm{Si}$ phase appears, and the granular $\mathrm{Mg} 2 \mathrm{Si}$ phase grows, causing some casting defects such as pores to occur, and the serration behavior in the alloy disappears, the mechanical properties of the alloy are reduced but higher than without Si addition (as shown in the Figure 4). Therefore, the mechanical properties of the alloy can be optimized under the critical state of the serration behavior of the alloy. The purpose of optimizing the mechanical properties of the alloy can be achieved by paying attention to the serrated flow behavior of the alloy, which provides a new idea for optimizing the mechanical properties of the alloy.

When the Si content reaches 0.9 at.\%, the stress-strain curve of the alloy tends to be smooth, and the stress drop is very small. In order to better study the relationship between serration 
behavior and alloy properties, we can use elastic strain energy instead of stress drop. The area of stress and strain in the stress rise curve on the stress-strain curve of the alloy reflects the elastic strain energy of the alloy, which is shown in Figure 7. We can find that the elastic strain energy of the alloy increases first and then tends to be stable as the strain increases. With the addition of $\mathrm{Si}$, the average elastic strain energy increases and the serration behavior becomes weaken. It is coincident with the compressive stress-strain curves, which were shown in Figure 5.

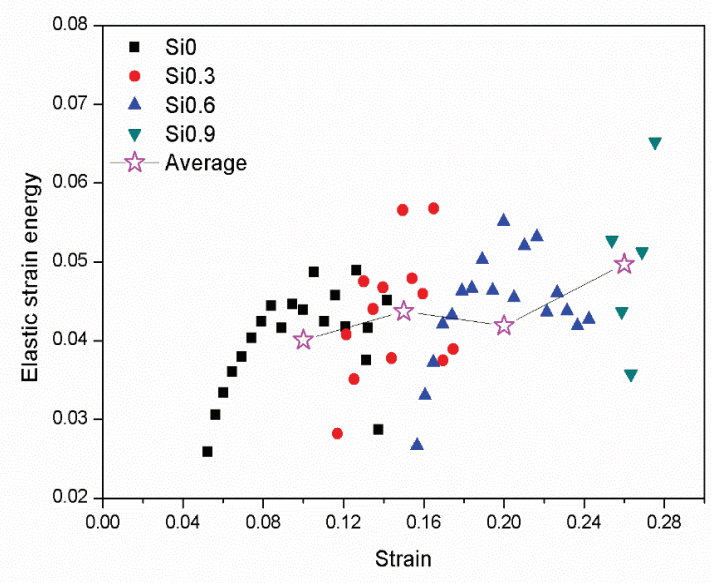

Figure 7 the elastic strain energy of the alloy with different $\mathrm{Si}$ addition

The analysis of the elastic strain energy of the alloy shows that the effect of precipitation on the elastic strain energy of the alloy is very obvious, which also affects the serration behavior. With the addition of $\mathrm{Si}$, the $\mathrm{Mg}_{2} \mathrm{Si}$ phase forms a new strengthening system with the original precipitation phase, which enhances the comprehensive mechanical properties of the alloy. When the amount of $\mathrm{Si}$ is added to 0.3 at.\%, the elastic strain energy of the alloy enhanced. When the Si content is further increased to 0.6 at.\%, the morphology of the precipitated phase changes, that is to say, the eutectic structure appears. As a result, although the content of precipitated phase has increased, the total strengthening effect has been weakened. In performance, the strength is improved, while the serration behavior is increased and the elastic strain is reduced. When the Si content reaches 0.9 at.\%, a large amount of eutectic $\mathrm{Mg}_{2} \mathrm{Si}$ and a small amount of granular Si-rich phase appear. The alloy get the highest compressive strength, the highest compression plasticity, and the improved elastic strain energy, with the serration behavior disappearing. Therefore, in addition to solid solution atoms, the morphology and type of precipitated phase also significantly affect the serration behavior of the alloy.

\section{Conclusions}

In this study, a series of light-weight $\mathrm{Al}(86-x) \mathrm{Mg} 10 \mathrm{Zn} 2 \mathrm{Cu} 2 \mathrm{Si} x$ medium-entropy alloys are successfully prepared, and the effect of $\mathrm{Si}$ content on the microstructure, properties and serration behaviors are investigated. The results are summarized as follows:

(1) Trace amounts of Si can significantly change the type and morphology of precipitated phases in the alloy, and the appearance of eutectic structure may improve the casting properties of the alloy.
(2) The addition of Si improves the mechanical properties of the alloy, when the Si content reaches 0.9 at.\%, the mechanical properties of the alloy are optimal.

(3) The serration behaviors of the alloys also change with the addition of Si. The mechanical properties of the alloy can be optimized under the critical state of the serration behavior, which indicats that the type and morphology of the precipitated phase can significantly affect the serration behavior of the alloy.

Author Contributions: The experiments and writing papers is by Yasong Li, and the paper discussion and revision was assist by Ruixuan Li. Yong Zhang was the corresponding author.

Conflict of Interest: No conflict of interest was reported by the authors.

Acknowledgement: The authors would like to thank the National Science Foundation of China (NSFC, Grants 51671020)and Dongguan Yian Technology Co., Ltd. for the financial support.

\section{References}

[1] Li H, Cao F, Guo S, et al. Effects of Mg and Cu on microstructures and properties of spray-deposited Al-Zn-Mg-Cu alloys[J]. Journal of Alloys and Compounds. 2017, 719: 89-96.

[2] G. M H, Jacobs, Spencer P J. Thermodynamic evaluations of the systems A1-Si-Zn and Cu-Mg-Ni[J]. Journal of Alloys and Compounds. 1995, 220: 15-18.

[3] Shu W X, Hou L G, Zhang C, et al. Tailored Mg and Cu contents affecting the microstructures and mechanical properties of high-strength Al-Zn-Mg-Cu alloys[J]. Materials Science and Engineering: A. 2016, 657: 269-283.

[4] Jung J, Park S H, Yu H, et al. Improved mechanical properties of Mg-7.6Al-0.4Zn alloy through aging prior to extrusion[J]. Scripta Materialia. 2014, 93: 8-11.

[5] Li Q, Zhao Y, Luo Q, et al. Experimental study and phase diagram calculation in $\mathrm{Al}-\mathrm{Zn}-\mathrm{Mg}-\mathrm{Si}$ quaternary system[J]. Journal of Alloys and Compounds. 2010, 501(2): 282-290.

[6] Li C, Sun J, Li Z, et al. Microstructure and corrosion behavior of $\mathrm{Al}-10 \% \mathrm{Mg} 2 \mathrm{Si}$ cast alloy after heat treatment[J]. Materials Characterization. 2016, 122: 142-147.

[7] Zhang Y, Zuo T T, Tang Z, et al. Microstructures and properties of high-entropy alloys[J]. Progress in Materials Science. 2014, 61: 1-93.

[8] Miracle D B, Senkov O N. A critical review of high entropy alloys and related concepts[J]. Acta Materialia. 2017, 122: 448-511.

[9] Zhang W, Liaw P K, Zhang Y. Science and technology in high-entropy alloys[J]. Science China Materials. 2018, 61(1): 2-22.

[10]Yan X H, Li J S, Zhang W R, et al. A brief review of high-entropy films[J]. Materials Chemistry and Physics. 2018, 210: 12-19.

[11]Cai Z, Cui X, Liu Z, et al. Microstructure and wear resistance of laser cladded Ni-Cr-Co-Ti-V high-entropy alloy coating after laser remelting processing[J]. Optics \& Laser Technology. 2018, 99: 276-281.

[12] Gao L, Liao W, Zhang H, et al. Microstructure, Mechanical and Corrosion Behaviors of CoCrFeNiAl0.3 High Entropy 
Alloy (HEA) Films[J]. Coatings. 2017, 7(10): 156.

[13]Yao Qiu, Sebastian Thomas, Mark A. Gibson, et al. Corrosion of high entropy alloys[J]. npj Materials Degradation. 2017(1): 1-18.

[14] Youssef K M, Zaddach A J, Niu C, et al. A Novel Low-Density, High-Hardness, High-entropy Alloy with Close-packed Single-phase Nanocrystalline Structures[J]. Materials Research Letters. 2015, 3(2): 95-99.

[15] Tseng K, Yang Y, Juan C, et al. A light-weight high-entropy alloy Al20Be20Fe10Si15Ti35[J]. Science China Technological Sciences. 2018, 61(2): 184-188.

[16]Feng R, Gao M C, Zhang C, et al. Phase stability and transformation in a light-weight high-entropy alloy[J]. Acta Materialia. 2018, 146: 280-293.

[17]Du X H, Wang R, Chen C, et al. Preparation of a LightWeight MgCaAlLiCu High-Entropy Alloy[J]. Key Engineering Materials. 2017, 727: 132-135.

[18]Li R, Gao J C, Fan K. Study to Microstructure and Mechanical Properties of Mg Containing High Entropy Alloys[J]. Materials Science Forum. 2010, 650: 265-271.

[19]Li R, Gao J C, Fan K. Microstructure and Mechanical Properties of MgMnAlZnCu High Entropy Alloy Cooling in Three Conditions[J]. Materials Science Forum. 2011, 686: 235-241.

[20]Yang X, Chen S Y, Cotton J D, et al. Phase Stability of Low-Density, Multiprincipal Component Alloys Containing Aluminum, Magnesium, and Lithium[J]. JOM. 2014, 66(10): 2009-2020.

[21]Baek E, Ahn T, Jung J, et al. Effects of ultrasonic melt treatment and solution treatment on the microstructure and mechanical properties of low-density multicomponent Al70Mg10Si10Cu5Zn5 alloy[J]. Journal of Alloys and Compounds. 2017, 696: 450-459.

[22]Ahn T, Jung J, Baek E, et al. Temperature dependence of precipitation behavior of $\mathrm{Al}-6 \mathrm{Mg}-9 \mathrm{Si}-10 \mathrm{Cu}-10 \mathrm{Zn}-3 \mathrm{Ni}$ natural composite and its impact on mechanical properties[J]. Materials Science and Engineering: A. 2017, 695: 45-54.

[23]Ahn T, Jung J, Baek E, et al. Temporal evolution of precipitates in multicomponent Al-6Mg-9Si-10Cu-10Zn-3Ni alloy studied by complementary experimental methods[J]. Journal of Alloys and Compounds. 2017, 701: 660-668.

[24]Li R, Wang Z, Guo Z, et al. Graded microstructures of Al$\mathrm{Li}-\mathrm{Mg}-\mathrm{Zn}-\mathrm{Cu}$ entropic alloys under supergravity[J]. Science China Materials. 2018.

[25] Hallstedt B. Molar volumes of Al, Li, Mg and $\mathrm{Si}[\mathrm{J}]$. Calphad. 2007, 31(2): 292-302.

[26] Liu Y, Zhang Y, Yu W, et al. Pre-nucleation clusters mediated crystallization in Al-Si melts[J]. Scripta Materialia. 2016, 110: 87-91.

[27] Sanchez J, Vicario I, Albizuri J, et al. Compound Formation and Microstructure of As-Cast High Entropy Aluminums[J]. Metals. 2018, 8(3): 167.
[28]Xu X, Yang Z, Ye Y, et al. Effects of various $\mathrm{Mg} / \mathrm{Si}$ ratios on microstructure and performance property of Al-Mg-Si alloy cables[J]. Materials Characterization. 2016, 119: 114-119.

[29] Tao G H, Liu C H, Chen J H, et al. The influence of $\mathrm{Mg}$ / $\mathrm{Si}$ ratio on the negative natural aging effect in $\mathrm{Al}-\mathrm{Mg}-\mathrm{Si}-$ $\mathrm{Cu}$ alloys[J]. Materials Science and Engineering: A. 2015, 642: 241-248.

[30]Li H, Cao F, Guo S, et al. Microstructures and properties evolution of spray-deposited Al-Zn-Mg-Cu-Zr alloys with scandium addition[J]. Journal of Alloys and Compounds. 2017, 691: 482-488.

[31] Guo M X, Zhang Y, Zhang X K, et al. Non-isothermal precipitation behaviors of Al-Mg-Si-Cu alloys with different Zn contents[J]. Materials Science and Engineering: A. 2016, 669: 20-32.

[32] Shao L, Zhang T, Li L, et al. A Low-Cost Lightweight Entropic Alloy with High Strength[J]. Journal of Materials Engineering and Performance. 2018.

[33] Mccormick P G, Ling C P. Numerical modelling of the Portevin-Le Chatelier effect[J]. Acta Metallurgica et Materialia. 1995, 43(5): 1969-1977.

[34]Zhang S, Mccormick P G, Estrin Y. The morphology of Portevin-Le Chatelier bands: finite element simulation for Al-Mg-Si[J]. Acta Materialia. 2001, 49(6): 1087-1094.

[35] Hutanu R, Clapham L, Rogge R B. Intergranular strain and texture in steel Luders bands[J]. Acta Materialia. 2005, 53: 3517-3524.

[36]Yanga F. Plastic flow in bulk metallic glasses: Effect of strain rate[J]. APPLIED PHYSICS LETTERS. 2007(91).

[37]Wei-Hua W. The nature and properties of amorphous matter[J]. PROGRESS IN PHYSICS. 2013, 33(5): 177-351.

[38]Chen S, Yang X, Dahmen K, et al. Microstructures and Crackling Noise of AlxNbTiMoV High Entropy Alloys[J]. Entropy. 2014, 16(2): 870-884.

[39]Chen S, Li W, Xie X, et al. Nanoscale serration and creep characteristics of Al0.5CoCrCuFeNi high-entropy alloys [J]. Journal of Alloys and Compounds. 2018(752): 464-475.

[40]Chen S, Xie X, Li W, et al. Temperature effects on the serrated behavior of an $\mathrm{Al} 0.5 \mathrm{CoCrCuFeNi}$ high-entropy alloy[J]. Materials Chemistry and Physics. 2018, 210: 2028.

[41]Zhang Y, Liu J P, Chen S Y, et al. Serration and noise behaviors in materials[J]. Progress in Materials Science. 2017, 90: 358-460.

[42] Kocks R A M F. New observations on the mechanisms of dynamic strain aging and of jerky flow[J]. Acta Metallurgica. 1979, Issue 7(Volume 27): 1125-1134.

[43] Cottrell A H. CRYSTALS, DISLOCATIONS AND PLASTIC FLOW[Z]. London: OXFORD AT THE CLARENDON PRESS, 1953134. 